\title{
Differential expression of placenta-specific 9 in cancers of the breast.
}

Shahan Mamoor, MS ${ }^{1}$

$3 \quad{ }^{1}$ shahanmamoor@gmail.com

East Islip, NY 11730

Breast cancer affects women at relatively high frequency ${ }^{1}$. We mined published microarray datasets ${ }^{2,3}$ to determine in an unbiased fashion and at the systems level genes most differentially expressed in the primary tumors of patients with breast cancer. We report here significant differential expression of the gene encoding placenta-specific 9, PLAC9, when comparing primary tumors of the breast to the tissue of origin, the normal breast. PLAC9 was also differentially expressed in the brain metastases of patients with metastatic breast cancer. PLAC9 mRNA was present at significantly lower quantities in tumors of the breast as compared to normal breast tissue. Analysis of human survival data revealed that expression of PLAC9 in primary tumors of the breast was correlated with distant metastasis-free survival in patients with luminal A subtype cancer, demonstrating a relationship between primary tumor expression of a differentially expressed gene and patient survival outcomes influenced by PAM50 molecular subtype. PLAC9 may be of relevance to initiation, maintenance or progression of cancers of the female breast.

Keywords: breast cancer, PLAC9, placenta-specific 9, systems biology of breast cancer, targeted therapeutics in breast cancer. 
Invasive breast cancer is diagnosed in over a quarter of a million women in the United States each year $^{1}$ and in 2018, breast cancer was the leading cause of cancer death in women worldwide ${ }^{4}$. While patients with localized breast cancer are provided a 99\% 5-year survival rate, patients with regional breast cancer, cancer that has spread to lymph nodes or nearby structures, are provided an $86 \% 5$-year survival rate $^{5,6}$. Patients with metastasis to distant sites, like the brain, are provided a $27 \% 5$-year survival rate ${ }^{5,6}$. Understanding how primary tumors are most transcriptionally different from the tissue from which they originate, the breast, can facilitate development of novel diagnostic and therapeutics to promote early detection and enhanced treatment, and contribute to efforts to prevent progression to metastatic stages. We mined published microarray data ${ }^{2,3}$ to understand at the transcriptome level and in an unbiased fashion genes most differentially expressed in primary tumors of the breast as compared to normal breast tissue. Placenta-specific 9 emerged as among the most differentially expressed genes in cancer of the female breast.

We utilized datasets GSE42568 ${ }^{2}$ and GSE100534 3 for this global differential gene expression

\section{Methods} analysis of female breast cancer. GSE42568 was generated using Affymetrix Human Genome U133 Plus 2.0 array technology with $n=17$ normal breast tissue biopsies and $n=104$ primary breast tumor biopsies from patients with breast cancer; analysis was performed using platform GPL570; the majority of patients whose tumors were analyzed were age 50 or older. GSE100534 was generated using Affymetrix Human Gene 1.0 ST Array with $n=16$ primary breast tumors and $n=3$ brain metastases from patients metastatic breast cancer; analysis was performed using platform GPL6244. The Benjamini and Hochberg method of $p$-value adjustment was used for ranking of differential expression but raw $p$-values were used to assess statistical significance of global differential expression. Log-transformation of data was auto-detected, and the NCBI generated category of platform annotation was used. A statistical test was performed to evaluate whether PLAC9 expression was significantly different between primary breast tumors and normal breast tissue using a two-tailed t-test. For Kaplan-Meier survival analysis, we used the Kaplan-Meier plotter online tool ${ }^{7}$ for correlation of PLAC9 mRNA expression levels with distant metastasis-free survival (DMFS) in $n=296$ patients with basal-like subtype cancer, $n=222$ patients with luminal A subtype cancer, $n=200$ patients with luminal B subtype cancer, $n=198$ patients with HER $2+$ cancer, and $n=27$ patients with normal-like subtype cancer, in the upper quartile.

\section{Results}

We performed discovery of genes associated with breast cancer in females by mining two independently published microarray datasets ${ }^{2,3}$.

\section{PLAC9 is differentially expressed in primary tumors of the breast.}

Studying the global gene expression profiles of 104 breast cancers from patients aged 31 to 89 revealed that the gene encoding placenta-specific 9, PLAC9, was among the genes most differentially expressed in tumors of the breast in human breast cancer (Chart 1). When sorting each of the genes expressed in tumors of the breast based on significance of difference as compared to normal breast tissue, PLAC9 ranked 77 out of 54675 total transcripts, equating to $99.9 \%$ differential expression (Chart 1). Differential expression of PLAC9 in female breast cancer was statistically significant (Chart 1; $p=3.16 \mathrm{E}-32$ ).

\section{PLAC9 is differentially expressed in brain metastasis in human breast cancer.}

Analysis of a second microarray dataset ${ }^{3}$, here studying global gene expression patterns in 3 brain 
metastatic tissues and 16 primary tumors of the breast, again revealed significant differential expression of PLAC9 in human breast cancer (Chart 2). When sorting each of the genes expressed in the brain metastasis of patients with breast cancer based on significance of difference as compared to normal breast tissue, PLAC9 ranked 77 out of 41093 total transcripts, equating to $99.8 \%$ differential expression (Chart 2). Differential expression of PLAC9 in the brain metastases of patients with breast cancer was statistically significant (Chart $2 ; p=3.16 \mathrm{E}-32$ ). These data suggested that differential expression of PLAC9 was not an artifact of a single microarray dataset, nor was it strictly associated with primary tumors of the breast, rather a general transcriptional feature representing a spectrum of transformation in human cancer..

\section{PLAC9 is expressed at significantly lower levels in breast tumors as compared to the breast.}

We obtained exact mRNA expression levels for PLAC9 from the breast and from breast tumors to

\section{PLAC9 expression correlates with survival outcomes in luminal A subtype human breast cancer.}

We performed Kaplan-Meier survival analysis to study relationships between tumor PLAC9 mRNA expression levels and survival outcomes in patients with breast cancer. We observed a correlation between PLAC9 expression and distant metastasis-free survival (DMFS) in patients with luminal A subtype breast cancer which trended towards statistical significance, in the upper quartile (Figure 2; $\log$ rank $p$-value: 0.091 for distant metastasis-free survival, hazard ratio: 0.42 (0.15-1.19) (Fig. 2)). PLAC9 mRNA levels were a positive prognostic indicator in luminal A subtype breast cancer patients. DMFS was, on average, markedly shorter for luminal A patients with low tumor expression of PLAC9 as compared to luminal A patients with high tumor expression of PLAC9 (Chart 3). PLAC9 primary tumor expression was not correlated with distant metastasis-free survival in basal-like breast cancer (Figure 2; $\log$ rank $p$-value: 0.36 for DMFS, hazard ratio: 1.27 (0.77-2.09) (Fig. 2)), luminal B subtype breast cancer (Figure 2; log rank $p$-value: 0.23 for DMFS, hazard ratio: 0.61 (0.27-1.38) (Fig. 2)), in HER2+ breast cancer (Figure 2; log rank $p$-value: 0.76 for DMFS, hazard ratio: 0.92 (0.53-1.59) (Fig. 2)) of in patients with normal-like breast cancer (Figure 2; log rank $p$-value: 0.69 for DMFS, hazard ratio: 0.65 (0.07-5.61) (Fig. 2)).

Thus, through comparative transcriptome analysis of primary tumors of the breast and normal breast tissue, we found that differential expression and down-regulation of PLAC9 was among the most significant transcriptional features in primary tumors from patients with breast cancer. PLAC9 expression in primary tumors of the breast was correlated with distant metastasis-free survival in patients with luminal A subtype disease, with mRNA levels of PLAC9 a positive prognostic indicator for luminal A breast cancer patients.

\section{Discussion}

Invasive breast cancer is a medical problem with a $27 \% 5$-year survival rate for women whose disease has spread to distant sites ${ }^{5,6}$. To facilitate understanding of the basic transcriptional differences between primary tumors of the breast and the tissues from which these tumors originate, normal breast tissues, we performed comparative transcriptome analysis using two independently published microarray datasets $^{2,3}$, providing evidence here that differential expression of placenta-specific 9, encoded by PLAC9, 


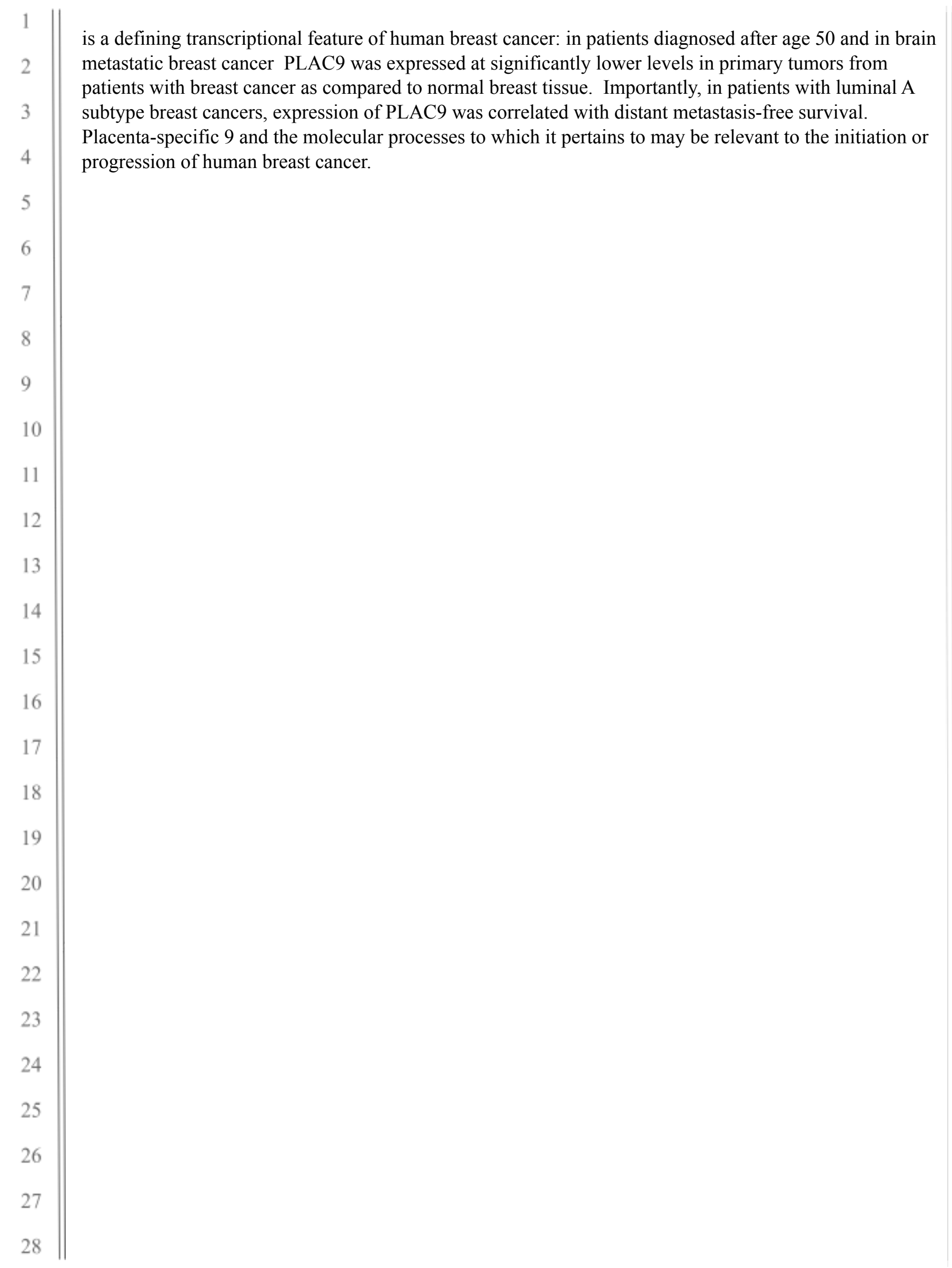




\section{References}

1. DeSantis, C.E., Ma, J., Goding Sauer, A., Newman, L.A. and Jemal, A., 2017. Breast cancer statistics, 2017, racial disparity in mortality by state. CA: a cancer journal for clinicians, 67(6), pp.439-448.

2. Clarke, C., Madden, S.F., Doolan, P., Aherne, S.T., Joyce, H., O’driscoll, L., Gallagher, W.M., Hennessy, B.T., Moriarty, M., Crown, J. and Kennedy, S., 2013. Correlating transcriptional networks to breast cancer survival: a large-scale coexpression analysis. Carcinogenesis, 34(10), pp.2300-2308.

5. Schulten, H.J., Bangash, M., Karim, S., Dallol, A., Hussein, D., Merdad, A., Al-Thoubaity, F.K., Al-Maghrabi, J., Jamal, A., Al-Ghamdi, F. and Choudhry, H., 2017. Comprehensive molecular biomarker identification in breast cancer brain metastases. Journal of translational medicine, 15(1), p.269.

4. Bray, F., Ferlay, J., Soerjomataram, I., Siegel, R.L., Torre, L.A. and Jemal, A., 2018. Global cancer countries. CA: a cancer journal for clinicians, 68(6), pp.394-424.

5. ACS Cancer Facts \& Figures 2019.

https://www.cancer.net/cancer-types/breast-cancermetastatic/statistics.

6. Survival Rates for Breast Cancer. https://www.cancer.org/cancer/breast-cancer/ understanding-a-breast-cancer-diagnosis/breast-cancer-survival-rates.html

7. Györffy, B., Lanczky, A., Eklund, A.C., Denkert, C., Budczies, J., Li, Q. and Szallasi, Z., 2010. An online survival analysis tool to rapidly assess the effect of 22,277 genes on breast cancer prognosis using microarray data of 1,809 patients. Breast cancer research and treatment, 123(3), pp.725-731. 


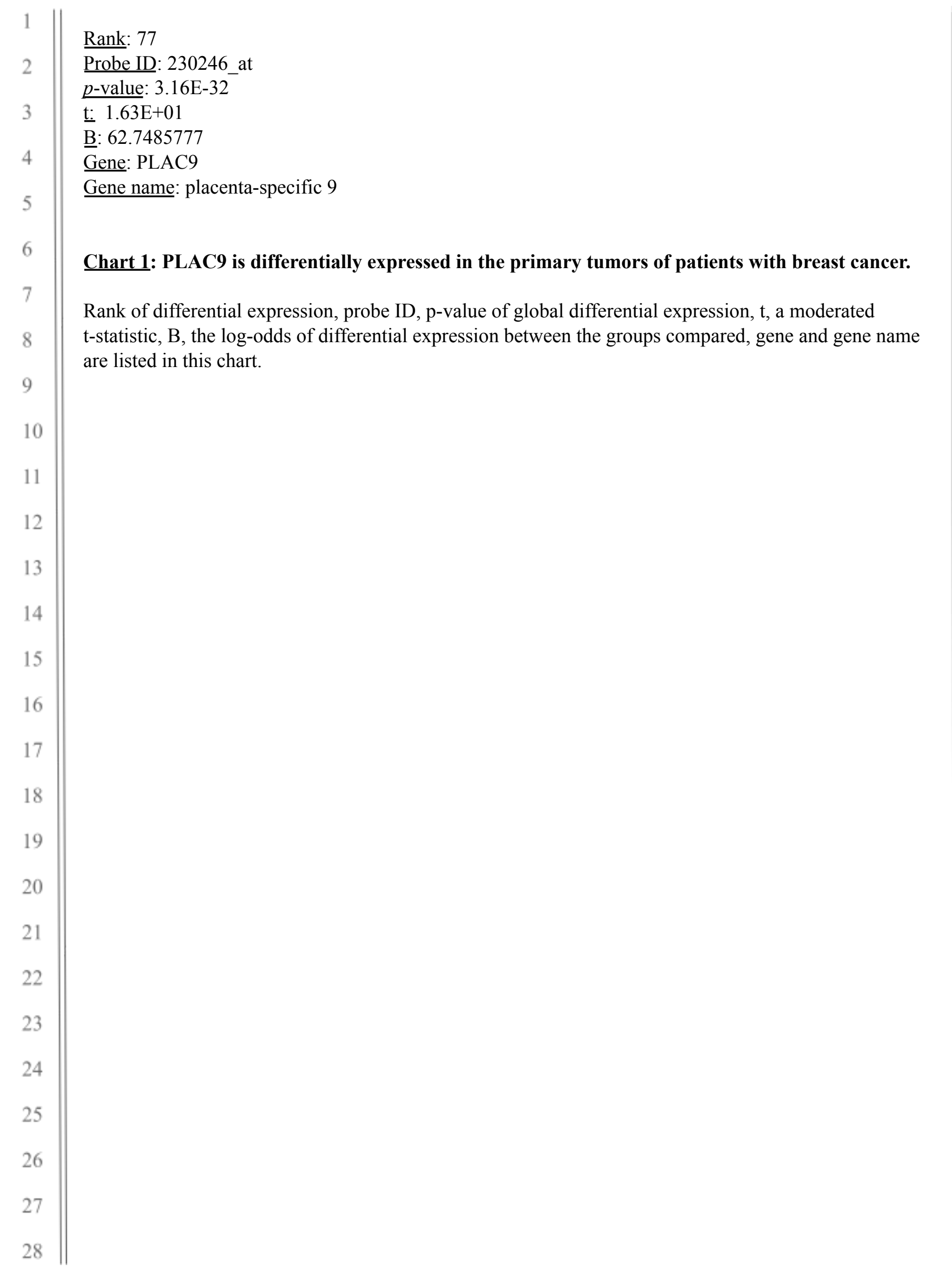




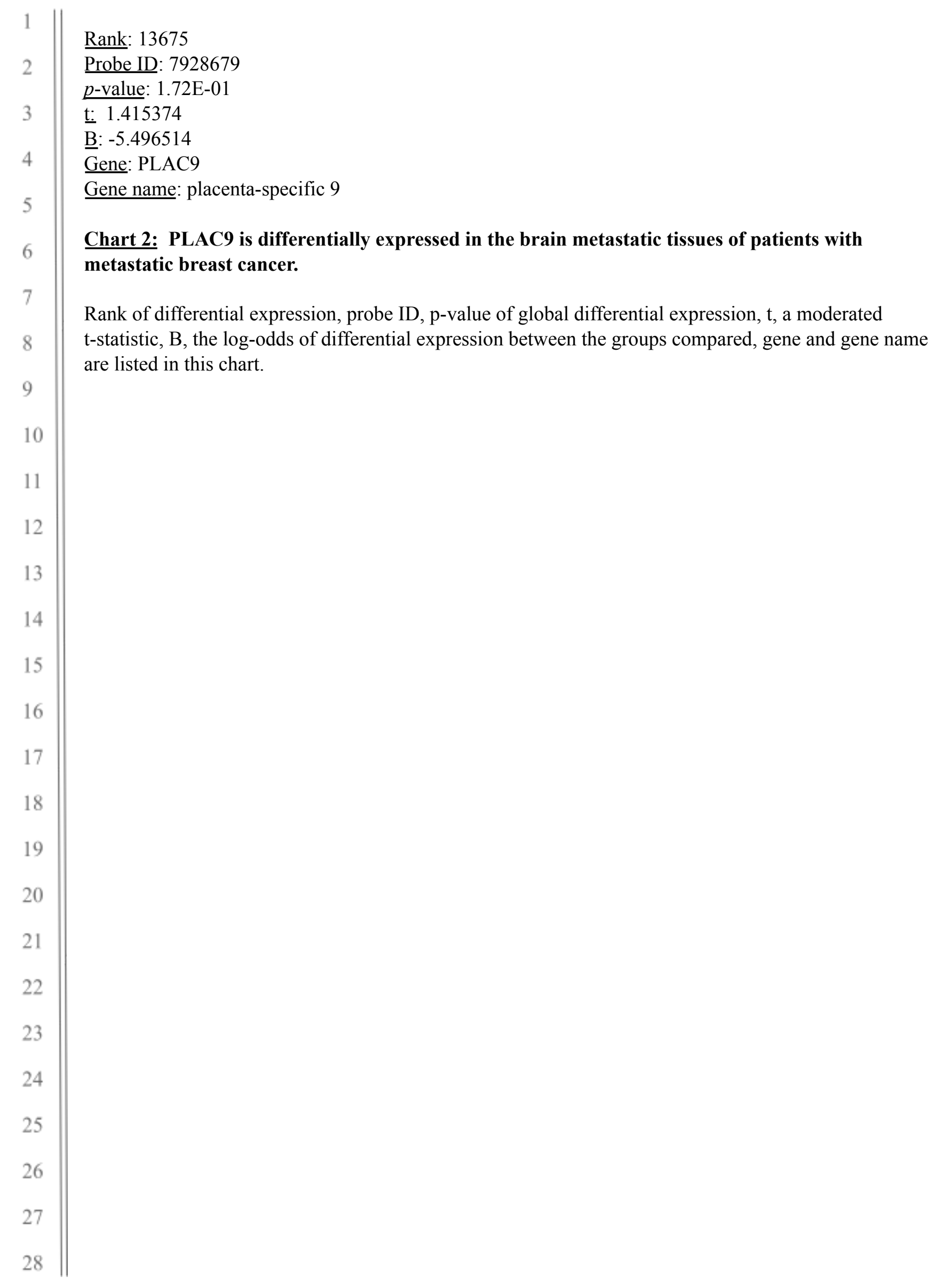




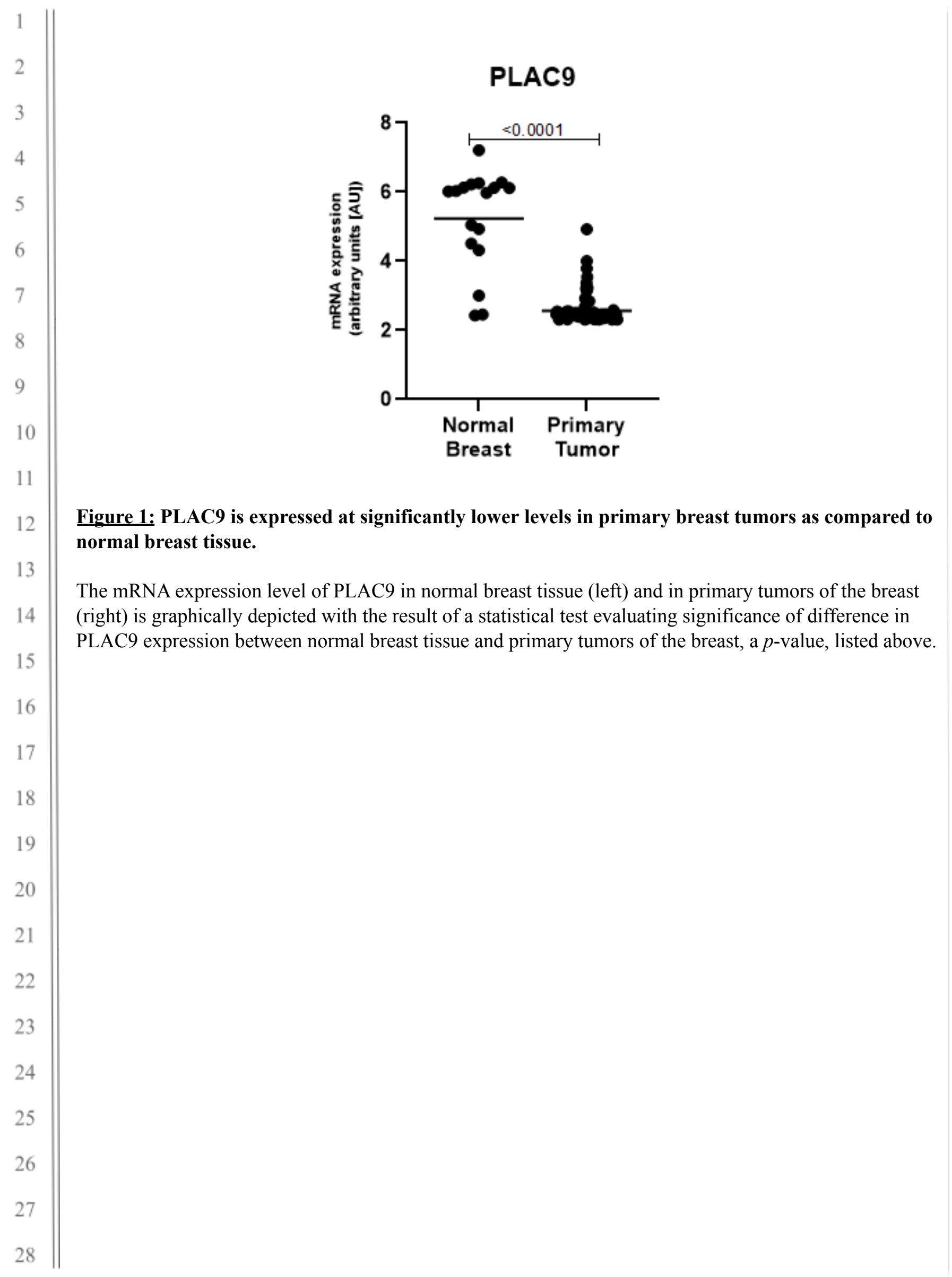




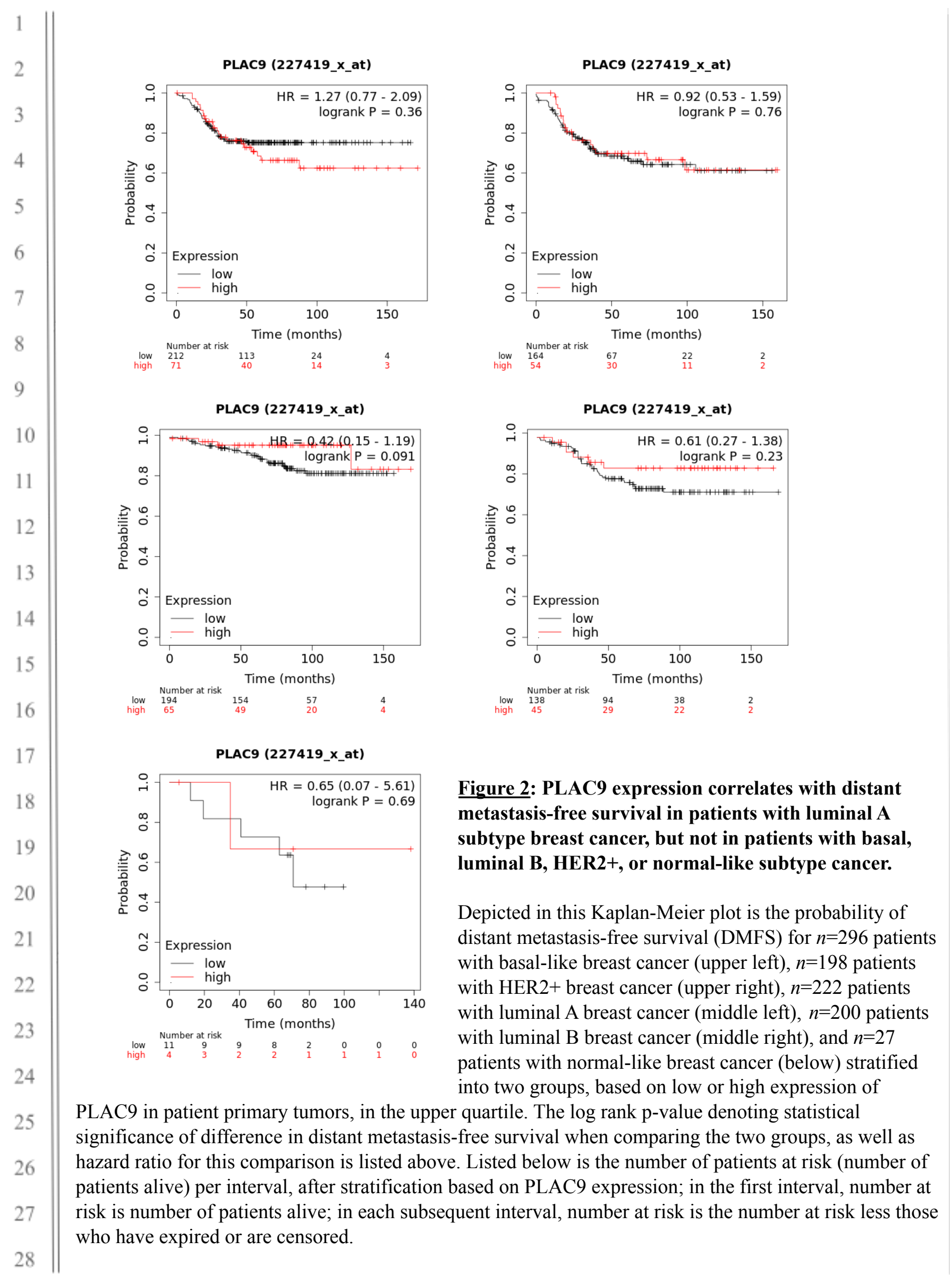

